VI

Leitungsverantwortung in der Krise 


\section{Der Umgang mit der Covid-19-Krise aus der Sicht der kollegialen Führung eines Universitätsklinikums}

Christa Tax, Gebhard Falzberger, Wolfgang Köle - befragt von Wolfgang Kröll

\section{Einleitung}

Das LKH-Universitätsklinikum Graz, welches im Eigentum des Landes Steiermark steht, ist einerseits ein Spital der Basisversorgung für den Großraum Graz südlich der Mur, andererseits Schwerpunktkrankenhaus für den Raum südlich der Mur-Mürz-Furche, die Zentralkrankenanstalt der Steiermark und Universitätsklinikum für den südösterreichischen Raum. Der Leitspruch „Menschen helfen Menschen“ wird im Klinikum Graz tagtäglich von den über 7.500 Mitarbeitern gelebt. Rund 1.500 Ärzte, 3.700 Pflegepersonen, über 1.000 Mitarbeiter des medizinisch-technischen und des sozialmedizinischen Dienstes sowie 1.300 Mitarbeiter der Verwaltung, Wirtschaft und Technik arbeiten Hand in Hand, um die medizinische Spitzenversorgung für über 1,8 Millionen Menschen im Einzugsgebiet des LKH-Universitätsklinikums Graz zu gewährleisten. Das LKH-Universitätsklinikum Graz versorgt pro Jahr an die 83.300 Patienten stationär und über 436.900 Patienten ambulant. Für die stationäre Betreuung stehen insgesamt 1.556 (systemisierte) Betten zur Verfügung, davon sind 175 Intensivbetten. In einem Jahr gibt es knapp 3.400 Geburten, damit gehört die Gebärklinik am Klinikum zu den größten im deutschsprachigen Raum. Jeden Tag bewegen sich 16.000 Personen am Gelände - Mitarbeiter und Mitarbeiterinnen, Patienten und Patientinnen, Besucher und Besucherinnen, Studenten und Studentinnen und Lieferanten. 19 Kliniken und zahlreiche Verwaltungsgebäude sind auf insgesamt 60 Hektar verteilt, davon ist ca. die Hälfte verbaute Fläche. Ein eigenes Straßennetz erstreckt sich über knapp zehn Kilometer, begleitet von 14 km Gehsteigen. Beim Energieund Wasserverbrauch ist das Klinikum vergleichbar mit der Stadt Hartberg.

Alle historischen Gebäude wurden 1912 im Jugendstil erbaut und über die Jahre durch moderne Gebäude, wie beispielsweise den 2017 eröffneten neuen Chirurgieturm, ergänzt. Unterhalb des Klinikums erstreckt sich ein unterirdisches Tunnelsystem auf drei Ebenen. Dank medizinischer Spitzenleistungen und höchster Qualitätsstandards hat sich das LKH-Universi- 
tätsklinikum Graz seinen ausgezeichneten Ruf bis heute bewahrt. Seit 2002 ist das Grazer Klinikum ein reines Universitätsklinikum und Ausbildungsspital der Medizinischen Universität Graz. Geleitet wird das LKHUniversitätsklinikum Graz einerseits durch das Direktorium des Hauses, bestehend aus der Pflegedirektorin DGKP Christa Tax, MSc, dem Betriebsdirektor Mag. Gebhard Falzberger sowie dem ärztlichen Direktor Ass.Prof. Dr. Wolfgang Köle. Da aber das LKH-Universitätsklinikum Graz nicht isoliert von der Medizinischen Universität Graz betrieben wird, werden bei Angelegenheiten, die beide Rechtsträger dieses Klinikums, nämlich die Steiermärkische Krankenanstaltengesellschaft m.b.H. und die Medizinische Universität Graz, betreffen, auch der Rektor und der Vizerektor der Medizinischen Universität involviert. Als Universitätsklinikum in Österreich und als Zentralklinikum der Steiermark nimmt das LKH-Universitätsklinikum Graz eine herausragende Stellung in der Behandlung und Betreuung der steirischen Patientinnen und Patienten sowie in bestimmten medizinischen Spezialgebieten auch für die Behandlung und Betreuung von Patienten benachbarter Bundesländer ein. Als enger Partner der Medizinischen Universität Graz ist das LKH-Universitätsklinikum Graz auch Ausbildungs- und Lehrkrankenhaus für Studierende der Medizin. Aufgrund der Größe des Krankenhauses - es zählt zu den größten Krankenanstalten Österreichs - und aufgrund der herausragenden Bedeutung für die Versorgung der steirischen Bevölkerung war es daher naheliegend, die Mitglieder des Direktoriums bezüglich ihrer Erfahrung sowie ihres Umgangs mit der aktuellen Pandemie zu befragen. 


\section{Fragen an Pflegedirektorin DGKP Christa Tax, MSc}

Kröll: Erinnerst du dich noch zurück, welche Gedanken dir durch den Kopf gingen, als du davon in Kenntnis gesetzt wurdest, dass aufgrund eines massiven Anstiegs an mit Covid-19 infizierten Menschen in deinem Verantwortungsbereich entsprechende Schutzmaßnahmen getroffen werden mussten?

Tax: Natürlich erinnere ich mich. Einige Wochen davor waren dieses Thema und die damit verbundene Infektionsgefahr für mich sehr weit weg und ein Problem in einem fernen Land. Und plötzlich war es ganz nah und auch bei uns angekommen. Es gab am Beginn natürlich viele Fragen und Unsicherheit. Von Anfang an war uns im Direktorium wichtig, die Mitarbeiterinnen und Mitarbeiter bestmöglich zu schützen. Wir haben in enger Zusammenarbeit und Abstimmung mit der Krankenhaushygiene und den Hygienefachkräften die erforderlichen Hygienemaßnahmen eingeleitet und räumliche Trennungen vorgenommen. Sehr rasch wurde als erste Maßnahme die Trennung an den Eingängen der Notaufnahmen von Patienten mit Symptomen und ohne Symptome durchgeführt. Patienten mit Symptomen wurden direkt vor dem Eingang von Mitarbeitern in voller Schutzausrüstung empfangen und gescreent und bei Verdacht auf eine Infektion in einem gesonderten Bereich weiterbehandelt. Rückblickend kann ich sagen, dass wir zum richtigen Zeitpunkt mit den Vorbereitungen und dem Planen von notwendigen Veränderungen begonnen haben.

Kröll: $\quad$ Was waren deine ersten persönlichen Gedanken im Umgang mit dieser drohenden Pandemie? Welche Schutz- und/oder Vorsichtsmaßnahmen hast du für dich selbst bzw. deine unmittelbaren Angehörigen getroffen?

Tax: $\quad$ Aus den vielen beruflichen Diskussionen und den Informationen war sehr schnell klar, dass es wichtig ist, die empfohlenen Maßnahmen strikt und konsequent auch in der familiären Umgebung einzuhalten. Die Informationen an die Bevölkerung seitens unserer Regierung waren sehr klar und verständlich. Die familiären Kontakte mit den Familienmitgliedern waren dann nur noch auf telefonische Kontakte reduziert. Das hat mich persönlich sehr betroffen gemacht, denn ein regelmäßiger Kontakt mit den Enkelkindern und den Eltern war mir immer sehr wichtig und bis zu diesem Zeitpunkt selbstverständlich. 
Kröll: Du trägst täglich für knapp 3.000 Mitarbeiter aus allen Pflegebereichen die Verantwortung. Einige davon werden um diese Zeit wohl auf Urlaub gewesen sein, möglicherweise auch im ferneren Ausland. Stand dir trotzdem ausreichend Personal zur Verfügung, um einen zu erwartenden Massenansturm an infizierten Personen betreuen zu können? Wie viele Pflegende hast du für die Betreunng von mit Covid-19 infizierten Menschen abstellen können bzw. müssen?

Tax: $\quad$ Obwohl für unsere Mitarbeiter aus der Pflege die Monate Februar und März beliebte Urlaubsmonate sind, war es nicht notwendig, Mitarbeiter aus dem Urlaub zurückzuholen. Da wir ab Mitte März mit unseren Leistungen heruntergefahren sind, war der zur Verfügung stehende Pool an Mitarbeitern aus der Pflege zu jedem Zeitpunkt der Krisensituation ausreichend groß, um die Situation zu bewältigen und die uns anvertrauten Patienten betreuen und versorgen zu können. Sehr wohl aber war eine Adaption der Dienstpläne erforderlich. Diese notwendigen Änderungen wurden mit großer Bereitschaft und Akzeptanz mitgetragen.

Kröll: Hast du Umverteilungen deiner Mitarbeiter vornehmen müssen, um ausreichend Pflegekräfte für die Betreuung infizierter Menschen zur Verfügung stellen zu können? Falls ja, wie wurde das von deinen Mitarbeitern angenommen? War die Bereitschaft gro $\beta$, sich in dieses unbekannte, gefährliche Milieu einzulassen?

Tax: Ja, es mussten laufend Umverteilungen vorgenommen werden, um Ausfälle z. B. wegen Absonderungsbescheiden und Quarantäneverpflichtungen kompensieren zu können. Es war auch notwendig, in den stark betroffenen Bereichen Personalverstärkungen vorzunehmen. Bedingt durch die notwendigen Schutzmaßnahmen und den hohen Pflegeaufwand der sehr kranken Patienten, mussten die Teams mit Personal aufgestockt werden. Gerade in den ersten Wochen mussten hier fast täglich Anpassungen vorgenommen werden. Die verantwortlichen Führungskräfte waren mit der Dienst- und Dienstplangestaltung sehr gefordert. Viele Kolleginnen und Kollegen mussten anderen Bereichen zugeteilt werden. Es war für mich beeindruckend, dass hier viel Verständnis und eine hohe Flexibilität bei den Kolleginnen und Kollegen vorhanden waren. Bei allen notwendigen Maßnahmen bestanden eine große Bereitschaft und ein unglaubliches Engagement seitens der Mitarbeiterinnen und Mitarbeiter. Natürlich gab es auch Ängste und Bedenken, war doch die gesamte Thematik für uns alle neu und völlig unbekannt. Die Führungskräfte haben hier 
wertvolle Arbeit in der Führung und Informationsweitergabe geleistet. Denn um die Ängste minimieren zu können, war es notwendig, viele Gespräche zu führen und die Sorgen und Bedenken ernst zu nehmen. Profitiert haben wir sicher davon, dass unser Ärztlicher Direktor Mitglied im Krisenstab des Unternehmens ist und hier eine enge Abstimmung und Beratung mit den Vorständen und der Geschäftsführung stattgefunden hat. Ergänzend dazu haben unsere Ärzte Informationen aus anderen Krankenhäusern im In- und Ausland eingeholt. Dadurch hatten wir auch die Gelegenheit, von diesen Erfahrungen unsere Vorgehensweise abzuleiten. So wussten wir zum Beispiel, dass wir im schlimmsten Fall mit einem Massenansturm von Intensivpatienten rechnen mussten, und konnten uns auf diese Eventualität vorbereiten.

Gemeinsam mit Führungskräften aus den Bereichen der Pflege, der Ärzteschaft und den technischen Betrieben wurde an einem Wochenende ein Plan für notwendige Veränderungen erarbeitet. Wir haben nicht nur die Notaufnahmen in die Versorgung für infizierte und nicht-infizierte Patienten getrennt, auch die stationären Versorgungsbereiche mussten entsprechend neu organisiert und umgestaltet werden. Dazu haben wir Covid-19-Verdachtsstationen, Covid-19-Stationen und Covid-19-Negativstationen auf den Universitätskliniken für Chirurgie, Innere Medizin, Frauenheilkunde und Geburtshilfe und auch auf der Universitätsklinik für Kinder- und Jugendheilkunde sowie Kinder- und Jugendchirurgie installiert. Diese Aufteilung der Patientenströme hat sehr gut funktioniert, da es eine sehr klare Trennung der Patienten gab. Die Herausforderung war, dass für diese Vorgehensweise ein höherer Personaleinsatz notwendig war. Und die tägliche Arbeit auf diesen Stationen war für das eingesetzte Personal, bedingt auch durch das Tragen der vollen Schutzausrüstung über mehrere Stunden, sehr anstrengend. Um Infektionen beim Personal möglichst zu vermeiden, war es wichtig, das richtige Handling und den korrekten Umgang mit der Schutzausrüstung gut zu schulen und zu sensibilisieren.

Durch das Lockdown haben wir nicht alle Mitarbeiterinnen und Mitarbeiter zur Behandlung und Betreuung vor Ort gebraucht. Hier gab es seitens des Unternehmens die Möglichkeit, Kolleginnen und Kollegen bei vollen Bezügen dienstfrei zu stellen. Sie mussten dabei aber jederzeit für einen notwendigen Einsatz zur Verfügung stehen. Damit haben wir Personalreserven geschaffen, auf die wir im Bedarf bei Ausfällen zurückgreifen hätten können. 
In dieser Ausnahme- und extrem belastenden Situation war es auch wichtig, bei der Diensteinteilung jener Mitarbeiter, die in kritischen Bereichen eingesetzt waren, ausreichende Erholungsphasen einzuplanen. Um all die Bereiche, die wir installierten, mit entsprechend ausgebildeten Mitarbeitern zu besetzen, wurden Mitarbeiter aus verschiedenen Kliniken zu den jeweiligen Hotspots zugeteilt, insbesondere an der Universitätsklinik für Chirurgie und der Universitätsklinik für Innere Medizin.

Kröll: Wie wurden in deinem Verantwortungsbereich Mitarbeiter fachlich über das Virus informiert? Haben sie Informationen darüber erhalten, wie mit dieser speziellen Situation umzugehen ist? Wie groß war die Angst der Mitarbeiter, selbst infiziert zu werden durch die Behandlung und Betreuung infizierter Patienten? Gab es ablehnende Haltungen und Einstellungen?

Tax: $\quad$ Mitarbeiter erhielten sowohl vom Rechtsträger als auch vom Direktorium regelmäßig schriftliche Informationen über die Ausbreitung und den Verlauf der Covid-19-Infektionen. Informationen, die ausschließlich einzelne Mitarbeiter betroffen haben, wurden diesen über persönliche Mails zur Verfügung gestellt. Zusätzlich wurden Informationen vom Krisenstab des LKH-Universitätsklinikums Graz, der täglich unter der Leitung des Ärztlichen Direktors stattfand, allen Führungskräften aktuell weitergegeben. Sie haben entschieden, welche Informationen für ihre jeweiligen Bereiche wichtig sind, und haben diese dann in persönlichen Gesprächen an die Mitarbeiter oder Teams weitergegeben. Im Direktorium wurde regelmäßig abgewogen, welche Informationen wichtig sind und wann und in welcher Form weitergegeben werden müssen. Die laufende, aber richtige Information ist gerade in Krisensituationen sehr entscheidend.

Hinsichtlich der Bereitschaft der Mitarbeiter im Umgang mit der Pandemie haben wir unterschiedliche Reaktionen erlebt. Zum Teil waren auch große Ängste vorhanden, oftmals bedingt durch medial verbreitete, zum Teil übertrieben dargestellte Informationen. Es sind immer wieder Fragen aufgetaucht und damit war eigentlich jeder Tag für die Führungskraft ein „Sich-Einlassen auf Unbekanntes“. Täglich stattfindende interdisziplinäre Krisensitzungen des Direktoriums habe ich persönlich als sehr wertvoll erlebt. Hier wurden die aktuellen neuen Informationen ausgetauscht, Fragen diskutiert und gemeinsame Vorgehensweisen fest- 
gelegt. Als Direktorium haben wir hier auch täglich einen guten Überblick zum Geschehen in den Kliniken bekommen.

Eine ablehnende Haltung gegenüber den Covid-19-Maßnahmen ist mir aus dem Pflegebereich nicht bekannt. Ich führe das zu einem großen Teil auf die täglich stattgefundene transparente Kommunikation und Information zurück. So habe ich Informationen aus der täglichen Krisensitzung an die Pflegeleitungen der einzelnen Abteilungen und Kliniken weitergegeben, bei der aktuelle Probleme und Herausforderungen diskutiert und das weitere Vorgehen besprochen wurden. Die Pflegeleitungen haben diese Informationen täglich an die Stationsleitungen weitergeben und diese dann an die Mitarbeiterinnen und Mitarbeiter. Um die Verbreitung des Virus einzudämmen, war es auch notwendig, Verhaltensregeln für die Zeit außerhalb der Patientenversorgung aufzustellen. So wurden zum Beispiel Regeln festgelegt, wie viele Personen sich in Sozialräumen etc. gemeinsam aufhalten dürfen, und eine Pausenregelung festgelegt, um wichtige Erholungsphasen sicherzustellen. In den Covid-19-Bereichen wurde das Essen (Früh, Mittag und Abend) sowie Mineralwasser für die Mitarbeiter direkt angeliefert und kostenlos zur Verfügung gestellt.

Kröll: Wie viele deiner Mitarbeiterinnen und Mitarbeiter sind an Covid-19 erkrankt? Waren das Mitarbeiter, die das Virus sozusagen aus einem Urlaubsgebiet eingeschleppt hatten, oder wurden die Mitarbeiter durch infizierte Patienten des Hauses angesteckt? Wie geht es diesen Mitarbeitern heute?

Tax: $\quad$ Es gab in meinem Bereich Mitarbeiter, die an Covid-19 erkrankt sind. Die Ansteckungen haben sich teilweise im familiären, teilweise im beruflichen Umfeld ereignet. Von Mitarbeitern, die sich diese Infektion während eines Urlaubs zugezogen hätten, ist mir nichts bekannt. Die relativ geringen Ausfälle durch Covid-19Erkrankungen waren gut zu kompensieren. Den Mitarbeiterinnen und Mitarbeitern geht es gut und sie sind mittlerweile wieder im Berufsalltag angekommen.

Kröll: Die Nicht-Verfügbarkeit bzw. die nur mangelhafte Verfügbarkeit von Schutzmaßnabmen wurde in Medien immer wieder angesprochen. Wie war es damit in deinem Verantwortungsbereich? Waren deine Mitarbeiter, die in die Betreuung von infizierten Patienten involviert waren, ausreichend geschützt? 
Tax: Der Schutz der Mitarbeiterinnen und Mitarbeiter war im Unternehmen immer die oberste Prämisse. Die Einkaufsabteilung und der Sicherheitstechnische Dienst am LKH-Universitätsklinikum Graz haben sich in enger Zusammenarbeit mit der Unternehmensführung und dem zentralen Einkauf intensiv bemüht, damit die erforderlichen Schutzmaterialien zur Verfügung stehen. Gleichzeitig wurde intensiv an Vorgaben für den richtigen Umgang mit der Krankenhaushygiene gearbeitet. Wir haben laufend Schulungen und Informationen für die Kolleginnen und Kollegen direkt auf den Stationen und in den Bereichen angeboten. Damit konnte sichergestellt werden, dass die Schutzausrüstung auch korrekt verwendet wurde. Ein möglicher Engpass an Schutzmänteln wurde im Unternehmen behoben, indem eine Alternative zu den Einmalschutzmänteln gefunden wurde. Anstelle der Einmalschutzmäntel wurden OP-Mäntel eingesetzt. Diese haben den großen Vorteil, dass sie einen viel angenehmeren Tragekomfort haben, wiederverwendet werden können und damit auch weniger Müll verursachen. Damit konnte der Engpass gut und sicher bewältigt werden. Bei einem weiteren Engpass bei den FFPSchutzmasken wurde ebenfalls nach einer Alternative im Unternehmen gesucht. Hier wurde schließlich die Methode der Vergasung und Aufbereitung der Masken angewendet. Mitarbeiter aus unserer Aufbereitungseinheit wurden für dieses Verfahren angelernt und so konnten wir allen Mitarbeitern die erforderlichen Schutzmasken zur Verfügung stellen. Die Ausgabe und Lieferung, inklusive des richtigen Einsatzes, waren zentrale Themen, die laufend je nach Situation angepasst wurden. Dank hohem Engagement in den unterschiedlichen Sparten, Kreativität und interdisziplinärer Zusammenarbeit, konnten Lieferengpässe gut überbrückt und die Sicherheit der Mitarbeiter die ganze Zeit über gewährleistet werden.

Kröll: Jetzt musste natürlich der Betrieb des Krankenhauses, wenn auch auf Sparflamme, so dennoch weiterlaufen. Wie lief das für die anderen Mitarbeiter - waren diese mehr oder weniger isoliert von jenen Pflegekräften, die sich der Betreuung von mit Covid-19 infizierten Patienten widmeten? Wie war es auf Intensivstationen - gab es welche, die ausschließlich für infizierte Patienten betrieben wurden?

Tax: Ja, wir sind in vielen Bereichen mit den Leistungen heruntergefahren, aber wir hatten auch Stationen, die sehr gefordert waren. Im Rahmen des Versorgungskonzepts des Unternehmens hat sich 
ergeben, dass das Klinikum den Auftrag der Akutversorgung im Großraum Graz, aber auch die Betreuung der Covid-19-positiven Patienten und der Verdachtsfälle, übernimmt, d. h., das Klinikum war eigentlich in jede Richtung gefordert und natürlich ist uns dabei auch entgegengekommen, dass in einigen Bereichen Leistungen reduziert worden sind. So hatten wir die Möglichkeit, in jene Bereiche Mitarbeiter zuzuteilen, wo zusätzliches Personal erforderlich war. Schon in der Planung der Krisenbewältigung spielten die Intensivstationen eine wesentliche Rolle. Es ging darum, zu organisieren, wie wir die Behandlung und Betreuung von Covid-19-Patienten ausrichten werden. Es gab dabei einen Stufenplan: Auf der Universitätsklinik für Chirurgie wurde eine Intensivstation als Covid-19-Intensivstation geführt, ein Teil der Intensivbetten der Universitätsklinik für Innere Medizin wurde getrennt und nur für Covid-19-Patienten zur Verfügung gestellt. Im Bereich der Universitätsklinik für Chirurgie wäre es möglich gewesen, noch weitere Intensivbetten als Covid-19-Intensivstationen zu führen. Das geplante volle Ausmaß an Intensivbetten wurde jedoch zu keinem Zeitpunkt benötigt. In den definierten Covid-19-Bereichen wurden eigene Teams zusammengestellt, die ausschließlich in diesen Bereichen arbeiteten. Es war eine große Herausforderung für die Führungskräfte, die Kolleginnen und Kollegen aus den unterschiedlichsten Stationen zu einem Team zusammenzuführen. In allen Bereichen ist dies bestens gelungen und ich bin sehr dankbar und stolz, dass alle zum Gelingen ihren persönlichen Einsatz und Beitrag geleistet haben.

Kröll: Nun scheint ja die Krise langsam wieder vorbei zu sein und alle sind dabei, die Systeme wieder hochzufahren. Wie läuft das in deinem Verantwortungsbereich? Lief der Betrieb, d. h. die pflegerische Versorgung der Patienten, wie eh und je?

Tax: Wie uns die derzeitigen Zahlen zeigen, ist die Krise weitgehend vorbei. Und wir hoffen auch, dass es nicht zu einer ,zweiten Welle“ kommt. Wir sind wieder schrittweise mit den Leistungen in den einzelnen Disziplinen hochgefahren. Wir als Direktorium haben dazu alle Kliniken besucht und hatten so die Möglichkeit, mit den Klinikleitungen die geplanten einzelnen Schritte zu besprechen und dabei auch die von uns erforderliche Unterstützung auszuloten. Es waren sehr interessante und informative Gespräche. Mit dem Rückgang der Anzahl von an Covid-19 erkrankten Patienten haben wir schrittweise die Covid-19-Bereiche wieder 
aufgelassen. Das dort eigens eingesetzte Pflegepersonal wurde auf das Vorliegen einer Infektion getestet, bevor es wieder in seine Stammbereiche zurückkehrte.

Kröll: Was hast du persönlich für dich, für dein privates Umfeld, aber auch für deinen Verantwortungsbereich innerhalb des Klinikums mitgenommen? Gibt es etwas, das du ändern möchtest oder das du ändern wirst?

Tax: Was ich für mich in meinen Verantwortungsbereich mitgenommen habe, ist, dass es sehr wichtig ist, die Mitarbeiter regelmäßig $\mathrm{zu}$ informieren, diese Informationen gut überlegt weiterzugeben und auf die Ängste, Bedenken und Bedürfnisse der Mitarbeiter einzugehen und zu evaluieren, was insgesamt machbar ist. Wichtig für meinen Bereich ist auch, den Kolleginnen und Kollegen zu vermitteln, dass man sie ernst nimmt, dass man für sie da ist, dass sie sich jederzeit an ihre unmittelbaren Vorgesetzten wenden können. Spannend war für mich auch, zu sehen, dass wir in kurzer Zeit Dinge geändert haben, worüber wir davor oft jahrelang erfolglos diskutiert hatten. Ganz besonders hat mir diese sehr fordernde und schwierige Zeit als Krankenhausleitung gezeigt, wie wichtig eine vertrauensvolle und gute Zusammenarbeit ist. Es ist wertvoll, dass anfallende Themen interdisziplinär rasch besprochen werden. Wichtig ist aber auch, dass es möglich ist, immer dann, wenn Fragen auftreten, diese kurzfristig im Direktorium zu besprechen, zu bewerten, Maßnahmen einzuleiten und diese dann zeitnah den Mitarbeitern zu kommunizieren. Dass ein derartiges Vorgehen erwünscht ist und sich positiv auswirkt, wurde mir auch wiederholt von den Mitarbeitern rückgemeldet. Durch die Miteinbindung und das tägliche Update in der Zeit des Lockdowns wurde ihnen das Gefühl vermittelt, dass sie gut durch die Krise geführt worden sind und sie sich so auf ihre Tätigkeit bei den Patienten konzentrieren konnten. Entscheidend für das Funktionieren der Versorgung von Patienten ist auch die enge und gute interdisziplinäre Zusammenarbeit. Ich möchte aber auch besonders die Zusammenarbeit mit den verantwortlichen Mitarbeitern der Betriebsorganisation hervorheben. Ambulanzen, Stationen und Bereiche wurden ausgesiedelt und es wurden eigene Covid-19-Bereiche gestaltet. Hier wurde nicht diskutiert, sondern alle Mitarbeiter waren hoch motiviert Hand anzulegen, wo sie gerade gebraucht wurden, und vieles wurde wirklich innerhalb kürzester Zeit realisiert. Dabei darf man nicht vergessen, dass Maßnahmen von Seiten der Logistik, der Wirtschaft und der Or- 
ganisation auf den ersten Blick oftmals gar nicht sichtbar waren, aber ein Betrieb ohne diese Schritte niemals möglich gewesen wäre. Wenn wir „dieses Miteinander“ aus der Krise heraus mitnehmen, dann sehe ich sehr positiv in die Zukunft dieses Krankenhauses und seiner Mitarbeiter.

Kröll: Wenn man jetzt zusammenfassend vom Worst-Case-Szenario ausgeht, dass noch einmal eine solche Pandemie auftritt, bevor ein Impsstoff dagegen entwickelt worden ist: Ist alles innerhalb deines Verantwortungsbereiches gut gelaufen, gibt es irgendeinen Bereich oder eine Situation, von der du sagen würdest, hier haben wir noch Verbesserungspotential? Oder andersherum: Standest du in Kontakt mit anderen Krankenanstalten innerhalb oder auch außerhalb der Steiermark und Österreichs und habt ibr euch bezüglich des Vorgehens in der Krise ausgetauscht? Falls das stattgefunden hat: Gab oder gibt es Ansätze, die du in deinem Verantwortungsbereich übernehmen würdest, bzw. haben jene Verantwortlichen, mit denen du Kontakt hattest, bekundet, Vorgehensweisen, wie sie in unserem Krankenhaus durchgeführt wurden, zu übernehmen bzw. zumindest auf ihre Verhältnisse zu adaptieren?

Tax: $\quad$ Mit meinen Kolleginnen und Kollegen nutze ich die nächste Zeit zur Reflexion. Wir werden die vergangene Thematik hinsichtlich Verbesserungspotentialen und Erfahrungen evaluieren und daraus abgeleitet die Maßnahmen und Gedanken verschriftlichen, um für etwaige zukünftige Situationen gerüstet zu sein oder bei einem neuerlichen Auftreten einer solchen Pandemie eine Stütze zu haben. Wichtig ist dabei für mich, dass diese Reflexion auf allen Ebenen sowie interdisziplinär stattfindet, denn nur so können wir einen umfassenden Rückblick gestalten. Während der Covid-19-Zeit wie auch in der Zeit davor habe ich meine Kontakte $\mathrm{zu}$ anderen Krankenanstalten innerhalb und außerhalb der Steiermark sowie international gepflegt. Auch wenn die Organisationen nicht eins zu eins vergleichbar sind, ergeben sich immer wieder interessante Gedankenanstöße und ein Austausch von Ideen und Erfahrungen. Und genau dieser Austausch ist zu jeder Zeit für beide Partner sehr gewinnbringend und wertvoll. 


\section{Fragen an Betriebsdirektor Mag. Gebhard Falzberger}

Kröll: $\quad$ Erinnerst du dich noch zurück, welche Gedanken dir durch den Kopf gingen, als du davon in Kenntnis gesetzt wurdest, dass aufgrund eines massiven Anstiegs an mit Covid-19 infizierten Menschen in deinem Verantwortungsbereich entsprechende Schutzmaßnahmen getroffen werden mussten?

Falzberger: Ja, ich kann mich noch sehr gut erinnern, als ein Schreiben vom Bundesministerium für Inneres bzw. vom Bundesministerium für Soziales, Gesundheit, Pflege und Konsumentenschutz eingetroffen ist, dass in allen Krankenanstalten Österreichs der derzeitige Betrieb massiv zu reduzieren ist, um entsprechende Vorhaltungen für Patienten, die mit dem Virus Covid-19 infiziert sind, zu treffen. Im ersten Moment waren wir, die Mitglieder des Direktoriums unseres Krankenhauses, aber auch meine engsten Mitarbeiter, verunsichert und betroffen, weil wir alle nicht wussten, was da auf uns zukommt.

Kröll: $\quad$ Was waren deine ersten persönlichen Gedanken im Umgang mit dieser drohenden Pandemie? Welche Schutz- und/oder Vorsichtsmaßnahmen hast du für dich selbst bzw. deine unmittelbaren Angehörigen getroffen?

Falzberger: Meine persönlichen Gedanken im Umgang mit der Pandemie sind mehrschichtig: Einerseits rede ich jetzt als Familienvater und Großvater, der während dieser Zeit leider keine Möglichkeit hatte, sein Enkelkind zu besuchen und zu sehen. Wir haben uns innerhalb der Familie an die Quarantänebestimmungen gehalten, mit betroffen war auch meine 87-jährige Mutter, die allein lebt. Ich merke aber jetzt auch, was diese Isolation bei vielen anderen Menschen bewirkt hat. Wir werden alle etwas Zeit brauchen, um wiederum in eine normale Kommunikation zu kommen, wie sie vor Ausbruch der Pandemie bestanden hat. Ich bin davon überzeugt, dass es sehr wichtig ist, dass wir wieder in unser Verhalten zurückkehren dürfen, wie es vor Corona gewesen ist.

Kröll: Das LKH-Universitätsklinikum Graz ist mit ca. 6.000 Mitarbeitern (ohne Med-Uni-Beschäftigte) der größte Arbeitgeber in der Steiermark. Innerbalb welchen Zeitraums konntest du überhaupt diese erforderlichen und vorgeschriebenen Schutzmaßnahmen installieren? Wie konntest du es gewährleisten, dass alle angeordne- 
ten Schutzmaßnahmen auch eingehalten wurden? Wie ist es dir und deinen Mitarbeitern überhaupt gelungen, diesen Betrieb nach außen so abzusichern, dass eigentlich nichts passiert ist und es zu keiner enormen Steigerung von Infektionen innerhalb der 6.000 Angestellten kommen konnte?

Falzberger: Für das LKH-Universitätsklinikum Graz war es eine sehr heikle Situation, da das Klinikum ursprünglich nicht primär als Krankenhaus für die Versorgung von mit Covid-19 infizierten Menschen vorgesehen war. Als sich das geändert hat, haben wir innerhalb kürzester Zeit den Betrieb der Krankenanstalt von etwa 1.500 Betten um mehr als 1.000 Betten reduziert, um die erforderliche Vorhaltung zu gewährleisten, sodass wir auch auf einen Ansturm infizierter Menschen gerüstet gewesen wären. Wir haben aber sehr rasch erkannt, dass in der Hochphase der Pandemie jeder Patient als potenziell infiziert zu behandeln ist. Das war eine sehr schwierige Situation und gleichzeitig waren wir, die verantwortliche Führungsebene, auch damit konfrontiert und herausgefordert, ob überhaupt ausreichend Schutzausrüstungen vorhanden sind bzw. der Nachschub für die erforderlichen Schutzausrüstungen gewährleistet ist. Es gab gerade zu Beginn auch sehr viele Probleme durch die Unberechenbarkeit des Virus. Oft wurden Patienten erst einige Tage nach der stationären Aufnahme positiv auf das Virus getestet. Im gesamten Klinikum wurden selbstverständlich alle Vorschriften, die von der Bundesregierung verfügt wurden, eingehalten und umgesetzt.

Kröll: Wie ist es dir gelungen, trotz der durchgeführten Schutzmaßnahmen den Betrieb dieses Unternehmens so aufrechtzuerhalten, dass alle Mitarbeiter und in der Folge auch alle Patienten mit den erforderlichen Gütern versorgt werden konnten?

Falzberger: Die Aufrechterhaltung des Betriebes ist nur durch den unglaublichen Einsatz extrem engagierter Kolleginnen und Kollegen gelungen. Wir als Direktorium des LKH-Universitätsklinikums Graz können stolz darauf sein, dass wir solche Mitarbeiterinnen und Mitarbeiter haben. 
Kröll: Welche Maßnabmen wurden seitens der Betriebsdirektion getroffen, sodass es beim An- und Abtransport von Gütern zu keiner Keimverschleppung - weder zu einer Verschleppung von Keimen in das Krankenhaus noch zu einer Verschleppung nach außen gekommen ist?

Falzberger: Sämtliche von den Experten empfohlene Maßnahmen für die Verhinderung einer Keimverschleppung wurden entsprechend eingehalten. Selbstverständlich hat sich der Desinfektionsmittelverbrauch am Klinikum während dieser Krisenzeit enorm gesteigert. Zudem haben wir auch versucht, outgesourcte Bereiche, wie z. B. den Bereich des Reinigungsdienstes, mit Schutzausrüstungen zu unterstützen.

Kröll: $\quad$ Tausende operative Eingriffe und Behandlungen mussten pandemiebedingt abgesagt bzw. auf einen späteren Zeitpunkt verschoben werden. Das führt aus betriebswirtschaftlicher Sicht zu einer enormen Umsatzeinbuße. Wie gehst du damit um? Wird dieser Verlust durch das Land Steiermark abgedeckt? Oder musst du irgendwo den Sparstift ansetzen? Und vor allem: Was bedeutet dieser Umsatzeinbruch für die Weiterführung des Um- und Neubaus der Universitätsklinik für Chirurgie?

Falzberger: Wir leben, Gott sei Dank, in Österreich und unser System der „leistungsorientierten Krankenanstaltenfinanzierung“ (LKF-System) ist anders organisiert als im europäischen Umfeld, sodass wir zumindest aufgrund der Absagen von operativen Eingriffen und Behandlungen kein finanzielles Problem haben. Wir hoffen, dass coronabedingte Verluste durch das Land Steiermark abgedeckt werden. Es ist unmöglich, überall den Sparstift anzusetzen. Hier muss eine Entscheidung getroffen werden, die unsere Generation für nachfolgende Generationen zu treffen hat. In erster Linie wird die gesamte Bevölkerung, aber auch die derzeitige und zukünftige Bundesregierung noch jahrelang, wenn nicht sogar jahrzehntelang, damit beschäftigt sein. Wir versuchen, unseren eigenen Bereich, aber auch insgesamt die steirische Spitalslandschaft so zu adaptieren, dass diese für möglicherweise auftretende ähnliche Krisensituationen künftig gut gerüstet sind. Wir haben festgestellt, dass nicht das Gesamtbettenproblem ein Thema ist, sondern dass es, falls überhaupt, im Bereich der Intensivbehandlungsbetten knapp werden könnte. Das spricht aus meiner Sicht dafür, dass die Gesundheitsre- 
form, wie sie in der Steiermark angedacht war, fortzusetzen ist. Ich hoffe und erwarte mir, dass die politischen Verantwortlichen die Kraft und den Mut aufbringen, weiter in Richtung Leitspitäler, wie insbesondere der Ennstal-Klinik, $\mathrm{zu}$ arbeiten.

Kröll: $\quad$ Gibt es unter deinen Mitarbeitern welche, die durch das Virus infiziert wurden? Falls ja, wie viele? Und vor allem: Wie geht es ihnen heute?

Falzberger: Es gab, Gott sei Dank, nur wenige Mitarbeiter aus meinem Verantwortungsbereich, die infiziert wurden. Es ist aber nicht feststellbar, ob sie sich im dienstlichen oder privaten Umfeld angesteckt haben. Jene fünf erkrankten Mitarbeiter aus meinem Bereich hatten im Wesentlichen keine großen Gesundheitsprobleme, vielmehr verlief die Infektion leicht. Ein Mitarbeiter wusste trotz der bestehenden Infektion nicht, dass er überhaupt erkrankt war. Die Bestätigung erhielt er viel später im Rahmen einer Routineuntersuchung.

Kröll: Nun scheint die Krise vorbei zu sein und der klinische Alltag beginnt von Neuem. Wie gehst du damit um? Gelingt es, den Klinikalltag wieder so herzustellen, wie er vor Corona war? Und wie weit bist du damit?

Falzberger: Wir, das Direktorium dieses Krankenhauses, konnten feststellen, dass das Herunterfahren des Krankenhausbetriebes einfacher war als das Wiederhochfahren. Die noch immer gültigen Abstandsregelungen führen dazu, dass es noch relativ lange dauern wird, bis wir wieder im Normalbetrieb arbeiten werden können. Wir mussten am Klinikum selbstverständlich auch die medizinische Versorgung der Menschen des Großraums Graz übernehmen. So war z. B. im Bereich der Universitätsklinik für Innere Medizin Vollbetrieb, sodass wir insgesamt am Klinikum eine "gemischte" Situation hatten.

Kröll: Wie sind aus deiner Sicht Mitarbeiter, Patienten und Besucher mit diesen Einschränkungen klargekommen? Gab es Probleme mit einer der Menschengruppen?

Falzberger: Sowohl Patienten und Besucher als auch Mitarbeiter sind aufgrund der Informationspolitik der Bundesregierung sehr gut informiert gewesen und alle haben für die getroffenen Maßnahmen großes Verständnis gezeigt. Jetzt aber kommen 
wir in eine Phase, in der es sehr viele medizinische Indikationen gibt, die einer entsprechenden Behandlung und Betreuung bedürfen. Jetzt machen sich natürlich Unruhe und Besorgnis breit, ob man als Patient dann tatsächlich zum OPTermin, zum Behandlungstermin oder zu einem erforderlichen Vorsorgetermin ins Krankenhaus kommen kann bzw. soll.

Kröll: $\quad D u$ hast an den Eingängen und Zufabrten zum Gelände Sicherheitspersonal aufstellen lassen. Welche Erfahrungen haben diese Mitarbeiter gemacht, insbesondere mit Menschen, die - aus welchem Grund auch immer - unbedingt in das Krankenhausgelände gelangen wollten?

Falzberger: Das Sicherheitspersonal, welches wir im und um das Klinikum positioniert haben, war retrospektiv betrachtet als Sicherheitsmaßnahme nicht unbedingt erforderlich, da die Disziplin der österreichischen Bevölkerung beispielgebend war. Aber es konnte anfangs niemand wissen, in welche Richtung sich diese Krise entwickeln wird. Sehr viele Menschen haben sich an die Ausgangsbestimmungen gehalten, es wurden am Klinikum wesentlich weniger Kontakte gezählt, die Inanspruchnahme bezog sich auf schwere und Schwerstfälle.

Kröll: Und was hast du persönlich für dich, für dein privates Umfeld, aber auch für deinen Verantwortungsbereich innerhalb des Klinikums mitgenommen? Gibt es etwas, das du ändern möchtest oder wirst?

Falzberger: Sowohl für mein privates Umfeld, aber auch für meine Tätigkeit innerhalb der Klinik habe ich gelernt, mittels Videokonferenzen perfekt zu kommunizieren. Aus meiner Sicht ist das eine Lösung für die Zukunft, die man jedenfalls andenken und weiterverfolgen muss. Es kann eigentlich auch nicht sein, dass man Dienstreisen von mehreren Stunden oder sogar über mehrere Tage tätigt, wenn eine Videokonferenz das gleiche Ergebnis erbringen kann. Es hat sich auch gezeigt, dass beispielsweise im Bereich des Controllings viele Mitarbeiter im Homeoffice ihre Arbeit ohne jeglichen Qualitätsverlust tätigen konnten. Ich werde jedenfalls den Bereich Homeoffice in meinem Verantwortungsbereich weiter ausgestal- 
ten, um hier Möglichkeiten neuer Technologien optimal zu nutzen.

Kröll: Wenn man jetzt zusammenfassend vom Worst-Case-Szenario ausgeht, dass noch einmal eine solche Pandemie auftritt, bevor ein Impfstoff dagegen entwickelt worden ist: Ist alles innerhalb deines Verantwortungsbereiches gut gelaufen, gibt es irgendeinen Bereich oder eine Situation, von der du sagen würdest, hier haben wir noch Verbesserungspotential? Oder andersherum: Standest du in Kontakt mit anderen Krankenanstalten, innerhalb oder auch außerhalb der Steiermark und Österreichs, und habt ibr euch bezüglich des Vorgehens in der Krise ausgetauscht? Falls das stattgefunden hat: Gab oder gibt es Ansätze, die du in deinem Verantwortungsbereich übernehmen würdest, bzw. haben jene Verantwortlichen, mit denen du Kontakt hattest, bekundet, Vorgehensweisen, wie sie in unserem Krankenhaus durchgeführt wurden, zu übernehmen bzw. zumindest auf ibre Verhältnisse zu adaptieren?

Falzberger: Wenn man nun retrospektiv, im Wissen, wie sich nun alles entwickelt hat, die Gesamtsituation betrachtet, dann hätte man sicher in der einen oder anderen Situation anders entschieden, aber es ist immer so, dass man Entscheidungen zu einem bestimmten Zeitpunkt treffen und nach Vorgaben der bestehenden Informationen agieren muss. Für das Vorliegen der damals bestehenden Informationen, speziell am Klinikum, wurde hervorragend reagiert. Wir haben auch intensivsten Kontakt mit dem AKH Wien und dem Universitätsklinikum Innsbruck gehalten und haben auch mit einem internationalen Berater, der unserem Krankenhaus auch in Nicht-Coronazeiten zur Verfügung steht, die Entwicklung in Deutschland mitanalysieren lassen. Besonders gut gelaufen ist in meinem Verantwortungsbereich die ausgezeichnete Zusammenarbeit innerhalb des Direktoriums - es herrschte wirklich blindes Vertrauen zwischen uns. Dies ist auch Voraussetzung dafür, dass solche Krisensituationen bewältigt werden können. 
III. Fragen an den ärztlichen Direktor Ass.-Prof. Dr. Wolfgang Köle

Kröll: Erinnern Sie sich noch zurück, welche Gedanken Ibnen durch den Kopf gingen, als Sie davon in Kenntnis gesetzt wurden, dass aufgrund eines massiven Anstiegs an mit Covid-19 infizierten Menschen nun in Ihrem Verantwortungsbereich entsprechende Schutzmaßnahmen getroffen werden mussten?

Köle: Die ersten Gedanken, die mir am Beginn dieser Krisensituation durch den Kopf gingen, waren einerseits die Notwendigkeit, die Mitarbeiter des Klinikums zu schützen, und andererseits, den betroffenen Patienten zu helfen. Hellhörig gemacht hat mich natürlich die Tatsache, dass es sich bei der Covid-19-Infektion um eine neue, weitgehend unbekannte Infektion handelt und wenig Kenntnisse über den Ausbreitungsmodus verfügbar waren, obwohl diese Erkrankung ja schon Monate zuvor in China aufgetreten war.

Kröll: Was waren Ihre ersten persönlichen Gedanken im Umgang mit dieser drohenden Pandemie? Welche Schutz- und/oder Vorsichtsmaßnahmen haben Sie für sich selbst bzw. Ihre unmittelbaren Angehörigen getroffen?

Köle: Da ich zumindest in Graz alleinstehend bin, hat sich diese Frage persönlicher Vorsichts- und Schutzmaßnahmen bzw. Verhaltensweisen im Umgang mit Angehörigen in diesem Sinne nicht gestellt. Die von mir getroffenen Vorsichtsmaßnahmen haben sich daher auf Social Distancing beschränkt.

Kröll: Mit Covid-19 infizierte Patienten benötigen einerseits Normalbetten, andererseits aber, wenn auch in geringerem Ausmaß, Intensivbehandlungsbetten. Wie ist es Ihnen gelungen, trotz einer wahrscheinlich vorbestehenden, mindestens 90-prozentigen Auslastung der Intensivbetten ausreichend Behandlungs- und auch Beatmungsplätze zur Verfügung stellen zu können? Haben Sie Umstrukturierungen innerhalb der Kliniken bzw. Abteilungen vorgenommen?

Köle: Geht man von den Ausführungen des Krisenstabs des Landes Steiermark und jenem der KAGes aus - in denen ich ebenfalls vertreten war -, dann hätte unser Krankenhaus, zumindest theoretisch, niemals mit an Covid-19 erkrankten Patienten konfrontiert sein dürfen, denn in der Steiermark war ein Krankenhaus als Covid-19-Krankenhaus vorgesehen, nämlich das LKH Graz II mit den Standorten Graz West, Graz Süd und dem LKH Hörgas. In 
diesen Krankenhäusern wurden die chirurgische und die medizinische Abteilung für nicht-infizierte Patienten geschlossen und ausschließlich für mit Covid-19 infizierte Patienten frei gehalten. Aber man hat relativ bald erkannt, dass dies nicht so funktionieren konnte, wie es geplant war, denn die Patienten des LKH-Universitätsklinikums Graz, die sich ja bereits a priori in unserem Haus speziellen Behandlungen unterzogen hatten, waren auch weiterhin im Klinikum zu behandeln, nämlich auch dann, wenn sie sich denn tatsächlich mit Covid-19 infiziert hatten. Damit war im LKH-Universitätsklinikum Graz von Beginn der Krise an die Situation gegeben, dass sowohl nicht-infizierte als auch infizierte Patienten behandelt werden mussten. Das hat die Verantwortlichen selbstverständlich vor große Herausforderungen gestellt: Denn initial waren die Intensivbehandlungsbetten an der Universitätsklinik für Innere Medizin mehr oder weniger zu 100 Prozent mit Patienten belegt, die nicht infiziert waren, und dies mit den üblichen Erkrankungen, wie man sie auf einer Intensivstation einer Universitätsklinik für Innere Medizin vorfindet: Myokardinfarkte, Lungenversagen, Pneumonien etc. Diese Intensivbehandlungsbetten, zumindest 50 Prozent davon, mussten daher für infizierte Patienten freigemacht werden. Die anderen 50 Prozent der Intensivbehandlungsbetten wurden weiterhin für nicht-infizierte Patienten benötigt, die mit Myokardinfarkten etc. einer Behandlung zugewiesen wurden. Aber man hat auch sehr rasch erkannt, dass diese Anzahl an verfügbaren Betten unzureichend ist, und daher mussten auch von den Intensivstationen der Universitätskliniken für Anästhesiologie und Intensivmedizin sowie für Chirurgie Intensivbehandlungsbetten zur Verfügung gestellt werden. Das war insofern problemlos durchzuführen, als das Routineoperationsprogramm weitgehend reduziert wurde und daher auch eine entsprechende Anzahl an Intensivbehandlungsbetten für infizierte Patienten zur Verfügung stand. Diese Kooperation mit den einzelnen Universitätskliniken bzw. den zuständigen klinischen Abteilungen hat sehr gut funktioniert. Zusätzlich waren aber auch andere große Umstrukturierungen innerhalb des Klinikums erforderlich: So wurde die zentrale internistische Notfallaufnahme ausschließlich für infizierte Patienten zur Verfügung gestellt, während die zentrale Notaufnahme der Universitätsklinik für Chirurgie interdisziplinär mit den Universitätskliniken für Innere Medizin und Neurologie für nicht-infizierte Patienten geführt wurde. An der Universitätsklinik für Innere Medizin wur- 
den Stationen in solche umfunktioniert, die ausschließlich mit Covid-19 infizierte Patienten aufnahmen, und auch an der Universitätsklinik für Chirurgie waren Umstrukturierungen erforderlich. Es wurden die Stationen der Klinischen Abteilung für Mund-, Kiefer- und Gesichtschirurgie der Universitätsklinik für Zahnmedizin und Mundgesundheit ausschließlich für infizierte Patienten frei gehalten, während die Patienten dieser Abteilung vorübergehend in eine freigemachte Station der Universitätsklinik für Hals-, Nasen- und Ohrenheilkunde verlegt wurden.

Kröll: Neuerungen in einem System bedeuten meist Widerstand. Wie rasch konnten Sie jene Klinikvorstände bzw. Abteilungsleiter, die in ibrem Bereich über Intensivbehandlungs- und Beatmungsplätze verfügen, so weit verpflichten, dass zumindest eine angemessene Zabl an solchen Betten verfügbar war?

Köle: Widerstände gegenüber den erforderlichen Maßnahmen hat es keine gegeben. Vielmehr haben alle Klinikvorstände und Abteilungsleiter unterstützend mitgearbeitet. Es war eher die Komplexität der Vorgaben von zuständigen Ministerien und anderen vorgesetzten Stellen, die zumindest zum Zeitpunkt der Anordnung vielleicht nicht immer logisch und nachvollziehbar durchdacht waren. Aber aus meiner Sicht liegt dies in der Natur der Sache, vor allem dann, wenn man es mit einer derart komplexen Situation zu tun hat.

Kröll: Mussten Patienten, die auf Intensivstationen bereits in Behandlung waren und bei denen absehbar war, dass diese Behandlungen noch längere Zeit in Anspruch nebmen würden, in andere Krankenanstalten verlegt werden, oder ist es gelungen, dennoch ausreichende Kapazitäten zur Verfügung zu stellen?

Köle: Grundsätzlich war es angedacht, Intensivpatienten in andere Krankenhäuser, in denen die Versorgung ebenfalls gewährleistet worden wäre, zu transferieren. Das hat aber nicht funktioniert, sodass wir auch diese nicht-infizierten Patienten an unseren Intensivstationen weiter behandeln mussten. Wir haben dies unter Aufwendung aller möglichen Schutz- und Vorsichtsmaßnahmen durchgeführt, vor allem mit dem Ziel, diese Patienten nicht zu gefährden und zu infizieren. Und wir können rückblickend feststellen, dass uns dies sehr gut gelungen ist. 
Kröll: $\quad$ Sie beschäftigen etwas mehr als 1.500 ärztliche Mitarbeiterinnen und Mitarbeiter (landesbedienstete Ärzte, aber auch Mitarbeiter der Medizinischen Universität Graz, die zur Patientenversorgung zugeteilt sind). War es möglich, alle zu schützen, d. h., hatten Sie ausreichend Schutzanzüge und Masken zur Verfügung? Falls nein, wie sind Sie damit umgegangen?

Köle: Beim Ausbruch der Infektion waren Masken und Schutzanzüge nicht in ausreichender Menge verfügbar - wobei aber auch die Wünsche der Mitarbeiter im Hinblick auf den erforderlichen Bedarf sehr individuell gestaltet waren und diese nicht immer den Empfehlungen der Fachärzte für Hygiene bzw. der Fachärzte für Infektiologie entsprochen haben. Da waren auch erhebliche Auffassungsunterschiede zwischen einzelnen Mitarbeitern: Es gab solche, die sich komplette Schutzanzüge wünschten, andere waren der Meinung, ein Mund- und Nasenschutz mittels Maske sei bereits ausreichend. Aber wir haben das anlassbezogen gelöst, d. h. zumindest in jenen Bereichen, in denen infizierte Patienten behandelt worden sind, konnten die Mitarbeiter damit rechnen, dass sie alle erforderlichen Schutzmaßnahmen erhalten. Es sind schließlich auch ausreichend Schutzanzüge zur Verfügung gestanden. Es wurde aber sehr bald erkannt, dass diese Schutzanzüge für gewisse Tätigkeiten, wie z. B. die Kommunikation, aber auch die Auskultation, eher hinderlich sind. Aber grundsätzlich muss festgehalten werden, dass man aus der Erkrankung gelernt hat - auch wie mit dieser Thematik korrekt umzugehen ist. Dann haben sich auch die Begehrlichkeiten geändert und man hat sich wieder auf ein geordnetes Arbeitsumfeld besonnen. Aber überschießende Reaktionen der ärztlichen Mitarbeiter sind auch verständlich, wenn man an Bilder aus italienischen Krankenhäusern denkt.

Kröll: $\quad$ Haben sich ärztliche Mitarbeiter mit diesem Virus infiziert - während eines Urlaubsaufenthaltes bzw. während der Behandlung von infizierten Patienten innerhalb des Krankenhauses?

Köle: $\quad$ Auch in meinem Verantwortungsbereich haben sich Mitarbeiter infiziert, und zwar sowohl während der beruflichen Tätigkeit als auch im privaten Umfeld. Selbstverständlich auch im Urlaub, so z. B. beim Wintersport am Arlberg. Aber es gab auch die Situation in einem peripheren Standardkrankenhaus, wo Mitarbeiter, die auf einer Kreuzfahrt am Nil unterwegs gewesen waren, infiziert, jedoch ohne Symptome, zur Arbeit erschienen sind, nicht wissend, dass sie andere Mitarbeiter und Patienten gefährden 
könnten, und die haben, wie zu erwarten, andere Menschen im Krankenhaus infiziert und dieses Krankenhaus musste vorübergehend vollkommen vom Netz genommen werden.

Kröll: Wie wurden in Ihrem Verantwortungsbereich Mitarbeiter fachlich über das Virus informiert? Haben sie Informationen darüber erhalten, wie mit dieser speziellen Situation umzugehen ist? Wie groß war die Angst der Mitarbeiter, durch die Behandlung und Betreunng infizierter Patienten selbst infiziert zu werden? Gab es ablehnende Haltungen und Einstellungen?

Köle: Grundsätzlich wurden die ärztlichen Mitarbeiter von der Zentraldirektion sehr umfangreich über das Virus, den potenziellen Verlauf der Erkrankung und die möglichen Auswirkungen informiert. Ich bin überzeugt, dass der Umfang der Information beinahe zu groß war, sodass es vielen Führungskräften wahrscheinlich gar nicht möglich gewesen ist, alle Informationen zu verarbeiten. Es wurden auch seitens des Direktoriums Mails an die Mitarbeiter ausgesandt und als ärztlicher Direktor habe ich zweimal wöchentlich die bereichsleitenden Ärzte über den jeweiligen Stand der Pandemie und ihrer Bewältigung informiert. Ebenso wurden die Klinikvorstände und die Leiter der klinischen Abteilungen von mir mehrmals informiert. Aber auch die Mitarbeiter der Pflege und die dem Betriebsdirektor unterstellten Angestellten erhielten täglich entsprechende Informationen über den aktuellen Stand der Pandemie.

Kröll: Die Zeit des Shutdowns war ja auch die Zeit der Vortragstätigkeit einiger Ihrer Mitarbeiter an den verschiedensten Orten dieser Erde. Wie sind Sie damit umgegangen, dass Ihnen diese Mitarbeiter nicht zur Verfügung standen? Wie sind diese Mitarbeiter aus dem Ausland wieder nach Hause gekommen? Waren sie zu Hause unter Quarantäne oder waren sie für Sie sofort verfügbar und einsatzbereit?

Köle: $\quad$ Ärztliche Mitarbeiter, die zu Beginn der Krisensituation von einer Vortragstätigkeit zurück an ihren Arbeitsplatz kamen, wurden primär in häusliche Quarantäne geschickt und waren daher auch in der Versorgung von Patienten nicht einsetzbar. Sie waren auch verpflichtet, sich bei der Gesundheitsbehörde zu melden, bzw. sie wurden vom Ministerium dahingehend registriert, dass sie aus einem Gebiet kommen, in der die Infektion bereits ausgebrochen war. Wenn aber in bestimmten Bereichen einige infizierte Mitarbeiter ausfallen, insbesondere in sehr speziellen Bereichen, wie z. B. der Kardiotechnik, dann ist die Aufrechterhaltung der Ver- 
sorgung der Patienten gefährdet. In diesen speziellen Fällen hat die Behörde dann dahingehend Ausnahmeregelungen geschaffen, dass diese Mitarbeiter nicht 14 Tage in häuslicher Quarantäne bleiben mussten, sondern bereits früher, unter Einhaltung von speziellen Hygienemaßnahmen, an ihren Arbeitsplatz zurückkehren konnten.

Kröll: Tausende Operationen und Behandlungen wurden in den letzten Monaten nicht durchgeführt. Wartelisten waren schon vor Corona ein Problem, das es abzuarbeiten galt, und, soweit ich noch informiert bin, ist das ja auch ganz gut gelungen. Nun kommt es wieder zu einem massiven Stau - wie gehen Sie damit um? Gibt es längere Betriebszeiten in den diversen OP-Einheiten?

Köle: $\quad$ Der Rückstau von Patienten, die sich aufgrund der Pandemie keinen operativen Eingriffen bzw. keinen anderen medizinischen Behandlungen unterziehen konnten, ist erheblich; wobei keine Zahlen genannt werden können, weil man ja nur mit den Zahlen des Vorjahrs mit entsprechender statistischer Unsicherheit vergleichen kann. Grundsätzlich würde ich es auch befürworten, in den OP-Einheiten längere Betriebszeiten einzuführen; dies ist jedoch ein sehr personalintensives Vorhaben, d. h., man müsste mehr Personal einstellen, aber soweit ich informiert bin, fehlen dafür, zumindest zurzeit, die finanziellen Mittel. Aber selbst dann, wenn die Finanzierung gelingen würde, ist es fraglich, ob man in der kurzen zur Verfügung stehenden Zeit ausreichend qualifiziertes Personal finden würde, und dies betrifft ja nicht nur den ärztlichen, sondern auch den pflegerischen Bereich. Man darf aber auch nicht vergessen, dass aufgrund der noch immer geltenden Abstandsregelungen auch die ambulanten und tagesklinischen Bereiche betroffen sind und dass es - selbst wenn die Möglichkeit bestehen sollte, längere Betriebszeiten wahrzunehmen kaum möglich sein dürfte, wesentlich mehr Patienten einer Operation zuzuführen als vor Ausbruch der Pandemie. Wenn man aufholen möchte, müsste man die Betriebszeiten auf 100 Prozent erhöhen, d. h., man müsste die Operationssäle 16 Stunden pro Tag auslasten. Ich bin davon überzeugt, dass es hier noch entsprechende Vorstöße geben wird, aber diese müssten entweder von der Zentraldirektion oder überhaupt von den politisch Verantwortlichen, d. h. von der Gesundheitslandesrätin bzw. dem Ministerium für Soziales, Gesundheit, Pflege und Konsumentenschutz, unternommen werden. 
Kröll: In diesem Zusammenhang: In den Medien wurde in den letzten Wochen auch das Nebenbeschäftigungsverbot von Ärzten während dieser Krisenzeit diskutiert und kritisiert. Wie gehen Sie damit um? Finden Sie es richtig, diese Nebenbeschäftigungen zu verbieten? Oder wäre es eine Lösung, Patienten, die auf eine Behandlung warten müssen bzw. mussten, in Ordinationen von als Wablärzte tätigen Kollegen zu behandeln? Wäre es aus Ihrer Sicht auch eine Möglichkeit, operative Eingriffe von Kollegen dieses Hauses in Privatkrankenanstalten durchführen zu lassen, zumindest so lange, bis die Wartelisten wieder einigermaßen abgearbeitet sind?

Köle: Grundsätzlich ist aus meiner Sicht nichts dagegen einzuwenden, wenn Kolleginnen und Kollegen neben ihrer klinischen Tätigkeit in Ordinationen oder auch in Privatkrankenanstalten tätig sind. Aber man muss auch hinzufügen, dass in Zeiten des Shutdowns auch die Privatkrankenanstalten ihre Leistungen heruntergefahren haben. Auch diese Krankenanstalten hatten dieselben Auflagen wie alle anderen Kliniken und Krankenhäuser und haben daher nur dringliche Patienten bzw. Notfälle behandelt. Bezüglich Wahlarztordinationen: Aus meiner Sicht dürften die Warteräume in diesen Praxen nicht so gestaltet sein, dass man tatsächlich sehr viele Patienten untersuchen kann. Aber als ärztlicher Direktor des Klinikums ist es mir lieber, dass man Patienten, die auf einer Warteliste stehen, im LKH-Universitätsklinikum behandelt und betreut, anstatt dies in einer Privatkrankenanstalt zu tun.

Kröll: Wie erklären Sie Patienten, die bereits vor der Covid-19-Pandemie sehr lange auf einen operativen Eingriff warten mussten, dass es jetzt noch länger dauern wird? Wie geht man als Arzt damit um, dass solche Menschen entweder wesentlich längere Zeit an starken Schmerzen leiden müssen (ich denke dabei an orthopädische Operationen) oder dass sie sogar auf einer Warteliste versterben werden (ich denke dabei an Herzoperationen)?

Köle: In diesem Zusammenhang ist es nur möglich, die betroffenen Patienten dahingehend zu informieren, dass alles getan wird, um sie so rasch als möglich zu behandeln. Man kann die Patienten um Verständnis bitten und hoffen, dass sie dies akzeptieren. Die Krisensituation und damit die Verschiebung von Operationen und medizinisch erforderlichen Behandlungen ist ja nicht hausgemacht, sondern wurde von außen in unser Land, in unsere Stadt, in unser Krankenhaus hineingetragen. 
Kröll: War es aus Ihrer Sicht richtig, diese Maßnahmen zu setzen, oder hätte möglicherweise ein weniger restriktives Vorgehen ebenfalls zum gleichen Erfolg geführt?

Köle: Die Maßnahmen, die von der Bundesregierung getroffen und angeordnet wurden, waren korrekt. Es wurde meiner Meinung nach von allen Verantwortlichen korrekt gehandelt: seitens der Regierung, seitens der KAGes, aber auch seitens der Medizinischen Universität Graz. Sollte es wieder einmal zu einer solchen Krisensituation kommen, kann man nur empfehlen, vor allem was den Krankenhausbereich betrifft, mehr oder weniger in gleicher bzw. ähnlicher Weise bei der Bewältigung vorzugehen. Man hat aber auch sehr viel gelernt hinsichtlich der Bereitstellung von Schutzausrüstungen und Desinfektionsmitteln.

Kröll: Nun scheint ja die Krise langsam wieder vorbei zu sein und alle sind dabei, die Systeme wieder hochzufahren. Wie läuft das in Ihrem Verantwortungsbereich? Sind Sie aus ärztlicher Sicht wieder im Vollbetrieb oder sind Sie erst langsam dabei, wieder das gesamte Spektrum medizinischer Leistungen des Krankenhauses anzubieten?

Köle: $\quad$ Der Betrieb unseres Klinikums ist weitgehend hochgefahren, wir laufen bereits seit längerem im Vollbetrieb. Reduktionen ergeben sich selbstverständlich noch aus den einzuhaltenden Vorsichtsmaßnahmen in Ambulanzen, Wartebereichen etc., aber ich bin überzeugt, dass etwa Mitte Juni der Betrieb wieder vollständig hochgefahren sein wird.

Kröll: Und was haben Sie persönlich für sich, für Ihr privates Umfeld, aber auch für Ihren Verantwortungsbereich innerhalb des Klinikums mitgenommen? Gibt es etwas, das Sie ändern möchten oder ändern werden?

Köle: Da gibt es vieles, was ich für mich persönlich aus der Krise gelernt habe. Ein wichtiger Aspekt ist die Form der Kommunikation: Ich bin überzeugt, dass man an der Krisenkommunikation einiges verbessern kann. Ich bin auch überzeugt, dass eine Digitalisierungsinitiative stattfinden muss, teilweise auch schon stattfindet; ich bin überzeugt, dass das LKH-Universitätsklinikum die Rolle als Zentralkrankenhaus sehr gut wahrgenommen hat, aber eine weitere Stärkung benötigt, um in Zukunft selbstständiger agieren zu können. Möglicherweise haben die Vorstände und Abteilungsleiter eine andere Einstellung zu zentralen Vorgaben, damit sind aber nicht jene des Direktoriums des Klinikums gemeint. Die meisten Gedanken und Argumente, die von den Klinikvorstän- 
den gekommen sind, waren sehr wertvoll und mussten auch intern abgestimmt werden; da komme ich wieder auf die Kommunikation in der Krise zurück - da ist die Medizin ein wenig zu kurz gekommen. Es wurden sehr viele Vorgaben hinsichtlich der Hygiene und von Infektiologen gemacht, aber es gibt auch ausreichend Experten im LKH-Universitätsklinikum Graz, die man bei der Lösung dieser Fragen mit hineinnehmen hätte sollen und können.

Kröll: Wenn man jetzt zusammenfassend vom Worst-Case-Szenario ausgeht, dass noch einmal eine solche Pandemie auftritt, bevor ein Impfstoff dagegen entwickelt worden ist: Ist alles innerhalb Ibres Verantwortungsbereiches gut gelaufen, gibt es irgendeinen Bereich oder eine Situation, von der Sie sagen würden, hier haben wir noch Verbesserungspotential? Oder andersherum: Standen Sie in Kontakt mit anderen Krankenanstalten, innerhalb oder auch außerhalb der Steiermark und Österreichs, und haben Sie sich bezüglich des Vorgehens in der Krise ausgetauscht? Falls das stattgefunden hat: Gab oder gibt es Ansätze, die Sie in Ihrem Verantwortungsbereich übernehmen würden, bzw. haben jene Verantwortlichen, mit denen Sie Kontakt hatten, bekundet, Vorgehensweisen, wie sie in unserem Krankenhaus durchgeführt wurden, zu übernehmen bzw. zumindest auf ibre Verhältnisse zu adaptieren?

Köle: $\quad$ Ich bin überzeugt, dass man, falls es erneut zu einer derartigen Situation kommt, besser vorbereitet sein muss, zumindest innerhalb des Klinikums, hinsichtlich der stationären Versorgung, aber auch hinsichtlich der Behandlung von Patienten auf Intensivstationen. Wir benötigen aus meiner Sicht eine höhere Anzahl an Betten auf Intermediate Care Units, die derzeit jedoch nicht verfügbar sind; in diesen Fällen fehlt uns die Infrastruktur vollkommen. Aus meiner Sicht kann man eine Pandemie nicht im Spital bekämpfen, eine Pandemie muss außerhalb eines Krankenhauses bekämpft und besiegt werden. Ich sehe es auch kritisch, dass man ganze Krankenhäuser als Infektionsspitäler vorsieht. Denn infizierte Patienten könnten auch in Krankenanstalten einer niedrigeren Stufe als in einem Zentralkrankenhaus, also in Standardkrankenhäusern, behandelt werden.

Kröll: Vielen Dank an die Mitglieder des Direktoriums, dass Sie sich in dieser schwierigen und herausfordernden Zeit, in der Sie selbst mit Arbeit und Problemen zugeschüttet sind, dennoch die Zeit genommen haben, eine Vielzahl von Fragen zu beantworten! 